\title{
Needleless Electrospinning of PA6 Fibers: The Effect of Solution Concentration and Electrospinning Voltage on Fiber Diameter
}

\author{
Alexandra Aulova1, ${ }^{1}$ - Marko Bek ${ }^{1}$ - Leonid Kossovich ${ }^{2}$ - Igor Emri ${ }^{1,3}$ \\ ${ }^{1}$ University of Ljubljana, Faculty of Mechanical Engineering, Slovenia \\ 2 Saratov State University, Russian Federation \\ ${ }^{3}$ Footwear Research Center, Tomas Bata University, Czech Republic
}

Needleless electrospinning is the process of forming thin material fibers from the open surface of its solution or melt in a strong electrostatic field. Electrospun non-woven materials are used in various applications that require specific fiber diameters and pore size distributions. Fiber diameter depends on the properties of the polymer solution and manufacturing conditions. A needleless electrospinning process using the Nanospider setup was investigated using the commonly used polyamide 6 (PA6) solution in a mixture of acetic and formic acids. Polymer solutions with different polymer concentrations were characterized by viscosity, surface tension and electrical conductivity. An increase in polymer content in the solution resulted in the exponential increase of the solution viscosity, polynomial increase of electrical conductivity and had almost no effect on surface tension. The effect of the polymer concentration in the solution, as well as electrospinning voltage on fiber diameter and diameter distribution, was investigated using scanning electron microscopy images. The average fiber diameter linearly increases with the increased polymer concentration and also demonstrates an increase with increased electrospinning voltage, although less pronounced. Therefore, a change in the PA6 solution concentration should be used for the robust adjustment of fiber diameter, while changes in electrospinning voltage are more appropriate for fine tuning the fiber diameter during the process of needleless electrospinning.

Keywords: needleless electrospinning, nanospider, PA6, fiber diameter, solution concentration, voltage

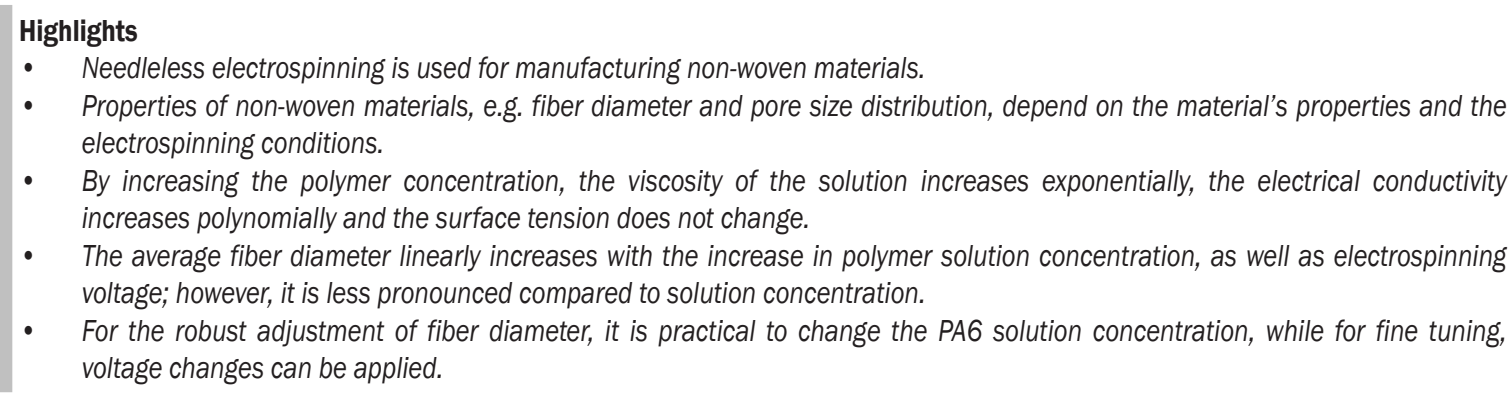

\section{INTRODUCTION}

Electrospinning is the process used to produce polymer fibers and membranes from a polymer solution or melt under high electrostatic forces.

Electrospun materials are used in various applications, including textiles [1], insulation materials, tissue scaffolds [2], patches, filters [3] and separation membranes [4]. The functionality of membranes for different applications is determined by the material, physicochemical surface properties of the fibers, fiber diameter and pore size distribution. Within this study, we investigated how the fiber diameter is affected by solution concentration and electrospinning voltage.

Together with membrane thickness, the fiber diameter determines the pore size distribution of the membrane. Fiber diameter determines the free surface of the membrane and pore size distribution determines the barrier and permeability properties of the material.
Fiber diameter and its distribution are strongly dependent on the polymer solution/melt properties and electrospinning conditions [5] and [6]. Controlling the fiber diameter during electrospinning allows for the formation of membranes with selected pore size distribution and is a crucial part of the electrospinning process.

The formation of electrospun fibers happens from the polymeric melt or solution between the two electrostatically charged electrodes. This process starts with the localization of the free charges in the polymer solution close to the surface in the electrostatic field [7]. As soon as the concentration of the charges is high enough, the surface of the solution forms the so-called Taylor cone [8] and [9]. The charge carriers continue to concentrate on the top of the cone, and this finally results in a jet of polymer solution being pulled by the electrostatic field towards the receiving electrode [7]. While the jet is travelling towards the receiving electrode, the solvent in the solution evaporates and 
the dry fiber is deposited. The process of solvent removal is governed by the properties of the solvent, the distance between electrodes and the environmental conditions during the electrospinning (temperature, airflow, humidity) [5] and [7].

There are three main properties of the polymer solution that are important for the electrospinning process: viscosity, surface tension and electrical conductivity. Their combination determines the behavior of the solution during electrospinning and directly affects the fiber diameter.

The viscosity of the polymer solution plays a crucial role in the electrospinning process as it affects the formation of the Taylor cone as well as the stabilization of the solution jet while travelling to the receiving electrode. During the first stage of fiber formation, high viscosity results in energy losses, but it also provides a more efficient process since more material is deposited. Additionally, a high enough viscosity of the polymer solution prevents the jet from breaking up into droplets and therefore stabilizes the jet [5] and [10].

Solutions with too high viscosity will not provide any fibers and solutions with too low viscosity will result in multiple droplets instead of a fiber (electrospraying).

Usually, viscosity is the parameter that significantly affects the electrospinning process and, consequently, the thickness of the electrospun fibers. Typically higher viscosity of the solution corresponds to the larger fiber diameters [5], [11] and [12]. The viscosity of the solution depends on the polymer concentration, its molecular weight and weight distribution, as well as molecular topology. It also depends on the loading conditions (frequency, shear rate) and environmental conditions (temperature, humidity), which are related to the electrospinning parameters.

Surface tension forces tend to reduce the surface of the liquid per mass unit. These forces are a result of interactions between solvent and polymer molecules (both topology and electromagnetic potentials). Surface tension forces (coupled with viscosity) are the main counterforces to the electrostatic forces that are pulling the fibers. Surface tension also determines the behavior of the fibers while forming and moving to the collecting electrode [5] and [12].

Electrical conductivity measures the ability of the material to carry an electrical charge and represents an intrinsic property of the solution. Conductivity mostly depends on the solvent's electrical conductivity [5], [12] and [13]. It is also affected by the polymer's molecular weight, polymer's concentration and temperature.

While surface tension and electrical conductivity can be changed using additives (surfactants or ionic salt), they also affect other properties and change the balance between surface tension, electrical conductivity and viscosity [11] and [14]. The empirical ranges of viscosity, surface tension and electrical conductivity required for successful electrospinning are defined, for example, in [5]; however, the range of values is relatively broad and can be used only in the needle electrospinning method. The needle or capillary electrospinning method is a process where one of the electrodes uses a needle through which the polymer solution/melt is pushed into the electrostatic field under pressure.

Needleless electrospinning, as its name suggests, does not use a needle or nozzle to introduce the polymer solution into the electrostatic field but instead uses a free surface or droplets of the solution on the electrode in the form of a rotating cylinder or string. Such a system has been introduced to the market under the commercial name Nanospider. The formation of fibers in this system occurs from the bottom rotating electrode to the top receiving electrode [15] and [16]. In this case, the process is more complex compared to conventional needle electrospinning, as the formation of Taylor cones happens not only on the surface of one polymer droplet but in multiple regions on the electrode. One critical advantage of this technology compared to nozzle technology is increased production capacity. Multiple fibers form at the same time without clogging the capillaries. Needleless technology also provides a more homogeneous electrostatic field and the possibility to produce largeer dimensions of electrospun material [15], [17] and [18].

The physics of needleless electrospinning process is different compared to the capillary method [5], [7] and [9]; as the Taylor cones form on a free surface subjected to electrostatic field, the shape of this surface is important. During capillary electrospinning, a Taylor cone forms on the droplet from the capillary, whos size is controlled by the pressure in the capillary. In the case of Nanospider technology, multiple Taylor cones appear on the surface of the thin film covering the cylinder electrode or droplets on the string electrode. The thickness of the polymer solution film and the size and density of droplets depend on the speed of electrode rotation and the solution's properties. In both cases, however, the solution's properties play the most crucial role in the formation of the electrospinning surface, as well as fiber dimensions and quality. Due to these differences 
in physics of the electrospinning process, a wellknown theory for capillary electrospinning cannot be directly applied to needleless fiber production technology. Differences in the shape of the solution's surface (a drop at the capillary or a drop on the string) and the interplay of multiple polymer solution jets in the electrostatic field during the material formation requires different settings and polymer concentrations for successful needleless electrospinning.

Within this work, the needleless electrospinning process of polyamide 6 (PA6) dissolved in a mixture of acetic and formic acids is investigated.

PA6 is a material widely used in a variety of applications including the automotive and textile industries, medicine and engineering. Its high mechanical strength in a wet and dry state [19], hydrophilicity and chemical resistance [20] make it an appropriate material for desalination, textiles [21], various filtration [22] and medical applications [23] water purification and gas separation. Additionally, PA6 can be modified with antibacterial/antifungal agents [24] and is often used in electrospun form for filtration, medical applications and textiles.

PA6 has been well studied using nozzle electrospinning [11], [14], [22] and [25] whereas, to the best of our knowledge, only limited research has been done using the Nanospider needleless methodology [21] and [24]. Researchers focused on the antibacterial additives to PA6 and morphological properties of the fiber's surface depending on the distance between electrodes [21] and [24]. There is a lack of information on the processing conditions of needleless electrospinning, which is essential for fiber properties and is the focus of this paper.

Within the paper, we conducted systematic research of the effect of polymer solution concentration on the critical properties of the polymer solution: its viscosity, surface tension and electrical conductivity. We investigated the effect of these parameters and the electrospinning voltage on the formation of PA6 fibers and their diameter. An electrospun material composed of fibers with the chosen diameter is the first step towards the production of materials with a controlled pore size distribution, as are required in different applications.

\section{EXPERIMENTAL METHODS}

\subsection{Materials}

Light-stabilized PA6 under the trade name Ultramid B24 N03 was provided through an in-kind donation by BASF (Germany). The material has a number average molecular weight (Mn) of 21,100 g/mol and a weight average molecular weight (Mw) of 55,600 g/mol. The material producer describes it as a material grade for the production of textile fibers that is especially suitable for high-rate spinning.

PA6 is dissolvable in the formic acid; therefore, for this study, a mixture of 2:1 by weight of acetic (AA, $100 \%$ glacial, EMSURE, Sigma-Aldrich, USA) and formic (FA, $99 \%$, Carlo Erba, France) acids were used to prepare the PA6 solutions. Both acids are weak carboxylic acids, which only partially dissociate in the solution. However, formic acid is a polar solvent with low polarity, which, in combination with PA6, results in the polyelectrolytic behavior of the solution [13]. This increases its electrical conductivity to values high enough for electrospinning [26]. In the mixture of acids, the formic acid acts as the aggressive PA6 solvent, while the presence of acetic acid increases the boiling point of the solvent $\left(118^{\circ} \mathrm{C}\right.$ for acetic acid and $108{ }^{\circ} \mathrm{C}$ for formic). This means that the jet of the solution during electrospinning will dry closer to the receiving electrode at the stage when the jet trajectory has already rotated in the normal direction to the receiving electrode [5]. This results in a higher product yield for Nanospider electrospinning technology.

\subsection{Preparation of Solutions}

The solutions for electrospinning and characterization were prepared following the same procedure. The components of the solution were mixed in the following sequence: polymer granulate, formic acid and acetic acid. Afterwards, the solution was mixed for approximately 8 hours at $200 \mathrm{rpm}$ using a magnetic stirrer. For mixing solutions with a larger content of the polymer, the magnetic stirrer was heated up to $50{ }^{\circ} \mathrm{C}$. Measurements were started 48 hours after the solutions were prepared.

The solutions were subjected to electrospinning in a laboratory setup three times. For each of the tests, a new batch of the solution was prepared following the same protocol. This was done in order to avoid the ageing of the solution and introducing the error of solution preparation into the whole process.

Due to the smaller amount of the solution prepared for characterization, the PA6 granulate 
was dried in order to avoid drastic errors in solution composition due to the polyamide water uptake. Drying was performed using a humidity analyzer (KE-DBS60-3, KERN, Germany) for 15 minutes at $80{ }^{\circ} \mathrm{C}$ using 10 grams of polymer granulate at once.

Five solution concentrations were prepared and their compositions are presented in Table 1.

Table 1. Solution compositions

\begin{tabular}{lccccc}
\hline Concentration [\%] & 11 & 12 & 13 & 14 & 15 \\
\hline PA6 mass [g] & 66 & 72 & 78 & 84 & 90 \\
\hline FA volume [ml] & 178 & 176 & 174 & 172 & 170 \\
\hline AA volume [ml] & 356 & 352 & 348 & 344 & 340 \\
\hline
\end{tabular}

\subsection{Characterization of Solutions}

\subsubsection{Viscosity}

Shear viscosity was measured using a rotational rheometer (MCR 302, Anton Paar, Austria) using a concentric cylinder in a cup. A cylinder with a $27-$ $\mathrm{mm}$ diameter and 1-mm gap between the cylinder and cup was used for measuring. To prevent solvent evaporation, we used a solvent trap.

Measurements were performed at ambient conditions by linearly increasing the shear rate from $11 / \mathrm{s}$ to $1501 / \mathrm{s}$ in $180 \mathrm{~s}$. Three repetitions per solution were made.

\subsubsection{Electrical Conductivity}

The electrical conductivity coefficient of the prepared solutions was measured using a Metrohm Conductometer 912 (Switzerland) equipped with a Pt1000 electrode (measuring ranges from 0.015 $\mathrm{mS} / \mathrm{cm}$ up to $250 \mathrm{mS} / \mathrm{cm}$ ), which determines the conductivity of liquid samples between the two platinum electrodes. Measurements were taken at ambient conditions six times for each of the solutions, after $120 \mathrm{~s}$ stabilization time.

\subsubsection{Surface Tension}

The surface tension coefficient was determined using a force tensiometer (K20, Kruss, Germany) equipped with the Wilhelmy plate, at ambient conditions. Measurements were performed 6 times for each of the solutions. The surface tension was determined as average over $10 \mathrm{~s}$ of measurement.

\subsection{Preparation of Electrospun Membranes}

The electrospinning process was performed using a Nanospider LAB (Elmarco, Czech Republic) laboratory setup and a string electrode. As a substrate, a polypropylene spunbonded material was used. The distance between electrodes was fixed to $140 \mathrm{~mm}$ and the speed of the substrate was set to $0.08 \mathrm{~m} / \mathrm{min}$.

All five solutions with different PA6 concentrations were electrospun at three voltage levels: $70 \mathrm{kV}, 75 \mathrm{kV}$ and $80 \mathrm{kV}$, respectively. The choice of this voltage range was made based on past experience using the Nanospider electrospinning setup as well as available research works [21], [24] and [25].

In total, 45 membrane samples were produced: 5 different electrospun solutions at 3 different voltages and 3 repetitions at each voltage level.

\subsection{Membrane Characterization}

All samples were investigated using a scanning electron microscope (SEM) with an autoemission cathode (MIRA II LMU, Tescan). For the SEM analysis, three samples were taken from each of the concentrations and voltage levels. Three different randomly selected places were selected at each sample, resulting in 9 testing areas per material. They were covered with $5 \mathrm{~nm}$ gold coating and analyzed using a SEM under $64.02 \mathrm{kx}$ magnification. For each location, 10 fibers were selected on the upper surface of the electrospun mat, and their diameters were measured, altogether resulting in 90 diameter measurements per each of the 15 samples types obtained via electrospinning.

The mean value of the fiber distribution was calculated, and the width of distribution was assessed using the uniformity coefficient [16] and [27], which is calculated similarly to the polydispersity index as the ratio of weight average, $A_{w}$ and number average, $A_{n}$, analogues:

$$
C_{F U}=\frac{A_{w}}{A_{n}},
$$

where

$$
A_{n}=\frac{\sum n_{i} d_{i}}{\sum n_{i}}, \quad A_{w}=\frac{\sum n_{i} d_{i}^{2}}{\sum n_{i} d_{i}},
$$

where $d_{i}$ is fiber diameter, and $n_{i}$ denotes the number of fibers with this diameter. A uniformity coefficient equal to 1 represents a perfectly monodisperse distribution of fiber diameter, while a higher number means broader fiber diameter distribution. 


\section{RESULTS AND DISCUSSION}

\subsection{Polymer Solution Properties}

Polymer solutions with different PA6 content levels were characterized in order to determine how polymer concentration affects viscosity, surface tension and electrical conductivity, see Fig. 1. A combination of these three properties determines if the solution will form fibers in an electrostatic field and the quality of fibers. Error bars were calculated as maximal errors for viscosity measurements and standard deviation for electrical conductivity and surface tension.

\subsubsection{Viscosity}

In the measured range of shear rates, up to $1501 / \mathrm{s}$ all materials exhibited Newtonian behavior. Within the measured shear rates, we did not observe shear thinning behavior, which is in line with findings in [28]. For the analysis, the viscosity was calculated as the average viscosity value in the measured shear rate range.

The results of average viscosity measurements as a function of PA6 concentration are shown in Fig. 1a. The viscosity increases with PA6 concentration. Comparing the viscosity of PA6 concentrations of 11 $\%$ and $15 \%$, there is an increase of $300 \%$.

The solutions are classified as concentrated [29] and demonstrate an exponential increase in viscosity with concentration (also demonstrated in [11], [14] and [25]). This increase can be evidence of an entangled regime of polymer chains [30]. Chain entanglements are the overlaps of the molecular chains in the solution, and a sufficient number of them is needed for the solution to form fibers without defects [31].

\subsubsection{Surface Tension}

The surface tension of the solution is a complex parameter that unites electrical and molecular interactions within the solution and is important for proper fiber formation. If the surface tension is too high, it will prevent the Taylor cone from forming or will result in the depositing of beads or droplets instead of fibers or the so-called electrospraying process. The higher the surface tension, the stronger the electrical field required to form fibers.

Fig. $1 \mathrm{~b}$ shows the surface tension coefficient of the tested solutions. The surface tension of the solution did not change significantly within the investigated range of polymer concentrations. The maximal variation of the average surface tension coefficient in respect of the solution with the smallest concentration is about $1.3 \%$. The error of surface tension measurement increases with polymer concentration, starting at $14 \%$. This might be attributed to the fast changes of solution properties due to evaporation, which is more pronounced in higher concentrations.
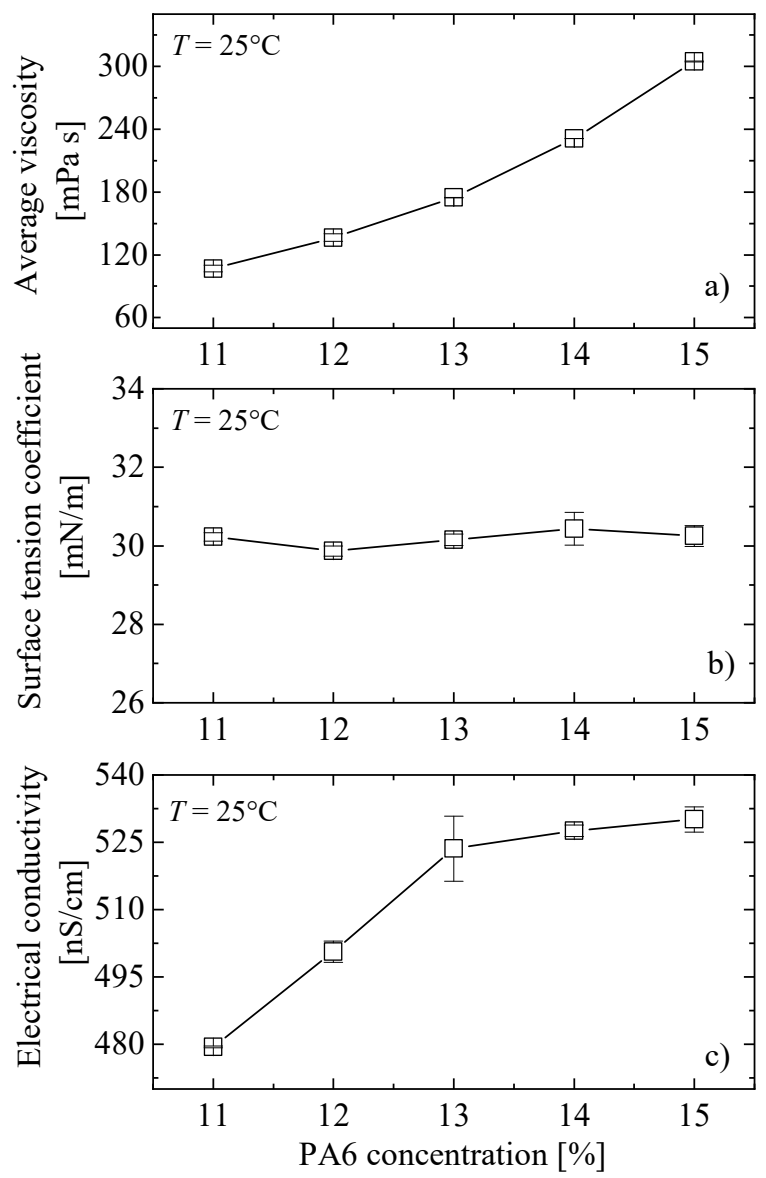

Fig. 1. Comparison chart of a) viscosity, b) surface tension, and c) electrical conductivity depending on PA6 concentration in the solution

In the literature, opposing findings on the effect of concentration solutions on surface tension can be found. On the one hand, researchers report an increasing surface tension with solution concentration [11] and [32]. However, in both cases, the investigation was done on much higher concentrations, up to $46 \%$ and $20 \%$ of Nylon- 4,6 and PA6 respectively. On the other hand, researchers report on a similar finding to ours. Guerrini et al. [33] reported that there was no significant influence of PA66 concentration on the surface tension coefficient. In their case, they investigated a concentration of between $10 \%$ and $20 \%$. 


\subsubsection{Electrical Conductivity}

In general, solution conductivity has a smaller role in fiber formation compared to viscosity and surface tension, but it can still affect the fiber size within 1 to 2 orders of magnitude [31].

Electrical conductivity measurements are shown in Fig. 1c and show an increase of the conductivity solution concentration up to $10.5 \%$ compared to the $11 \%$ solution.

Similar trends and behavior were observed by other researchers [11], [25], [32] and [34] in a comparable range of PA solutions. In a wider range of concentrations, researchers report an increasing electrical conductivity up to $16 \%$ followed by a drop [11], which might be related to the suppression of the charge carriers' mobility in more concentrated solutions.

Among all three tested solution parameters, viscosity shows the most pronounced change with the increase of concentration as it increases roughly by $300 \%$ compared to stable surface tension and a $10.4 \%$ increase in electrical conductivity.

Solution viscosity is considered to be a governing parameter affecting the electrospinning process also by several other researchers [11], [14] and [35]. Considering the findings of these researchers and our results, further analysis of the results will be done with respect to the polymer concentration, which is directly related the viscosity. However, the effect of increased electrical conductivity should not be diminished, as it results in an increased electrostatic force and plays a decisive role in forming fibers from more viscous solutions.

\subsection{Fiber Diameter and Distribution}

An analysis of fiber diameter distribution was done on 90 diameter measurements made for each concentration on the SEM image. One example per solution concentration is presented in Fig. 2.

Fig. 3 shows the analysis of fiber diameter depending on solution concentration and electrospinning voltage. Fig. 3 a shows the mean fiber diameter and Fig. 3b fiber uniformity index. Plotted error bars are calculated as the standard deviation of all fiber diameter measurements.

Fig. 3a demonstrates that polymer concentration almost linearly affects the mean value of fiber diameter and results in a maximum of $380 \%$ increase
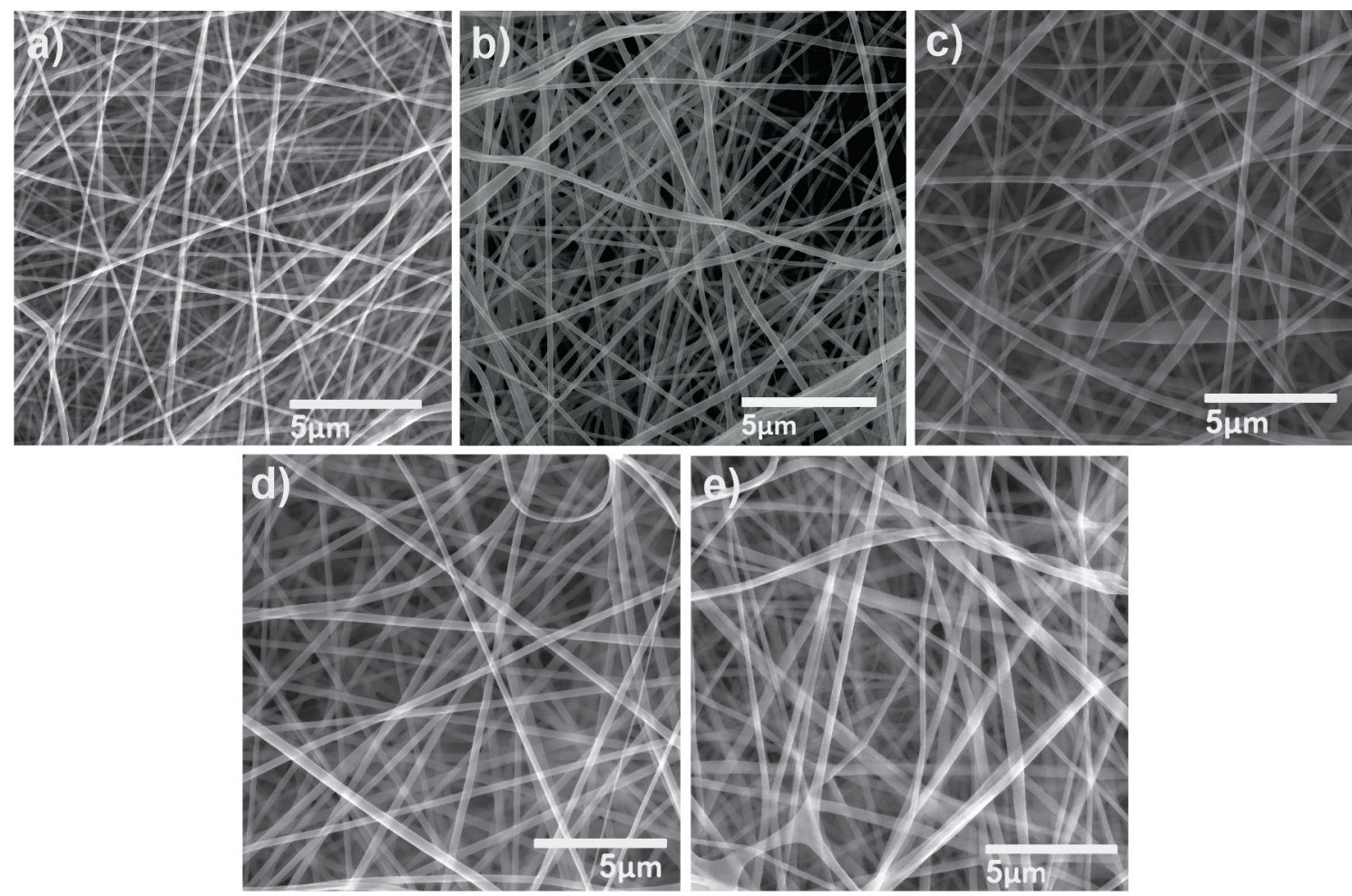

Fig. 2. SEM images of the membranes obtained from the solution of different concentrations at $75 \mathrm{kV}$ electrospinning voltage; a) $11 \%$ PA6, b) $12 \%$ PA6, c) $13 \%$ PA6, d) $14 \%$ PA6, and e) $15 \%$ PA6 

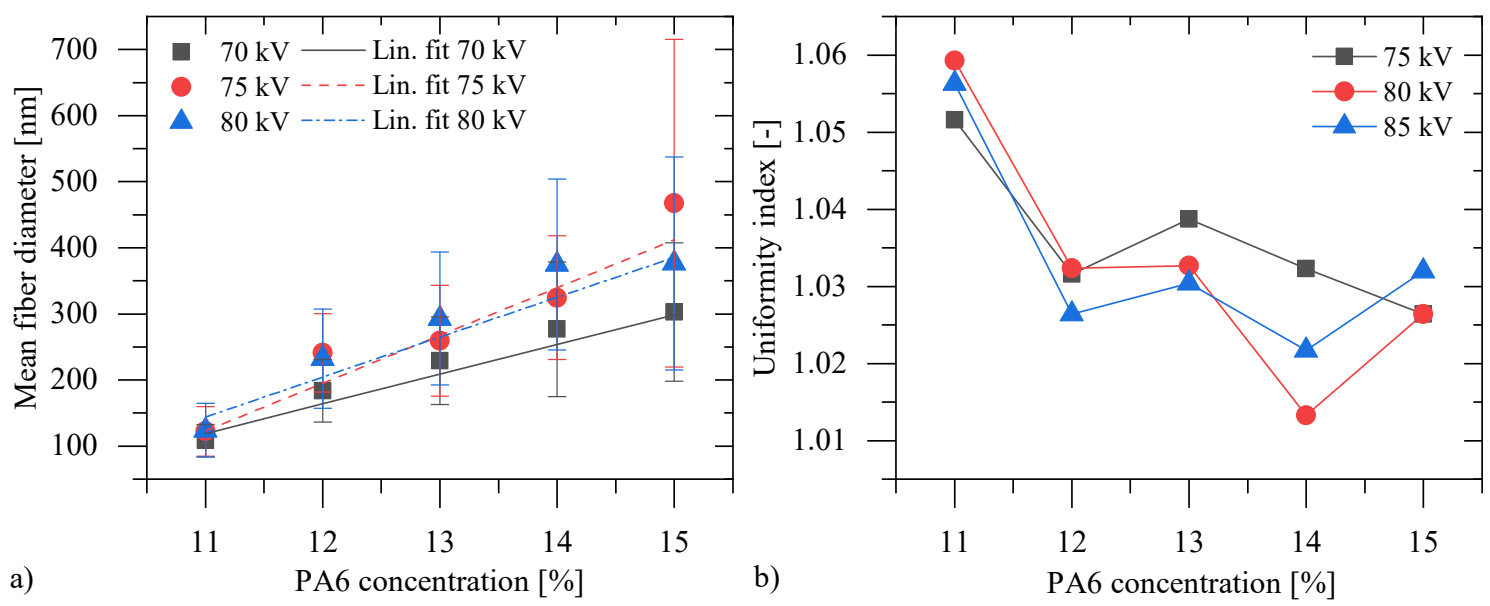

Fig. 3. a) Mean value of fiber diameter in respect of the polymer solution concentration for different electrospinning voltages, and b) uniformity index in respect of the polymer solution concentration for different electrospinning voltages

in fiber diameter for $75 \mathrm{kV}$. Error bars also increase with increasing polymer concentration.

The effect of voltage is negligible in the polymer solution with the lowest concentration but becomes more pronounced in higher concentrations (14\%). With an increase of voltage, the behavior of fiber diameter becomes less stable (larger error bars) and less linear (smaller $R^{2}$ value), which is probably related to the increase of Taylor cone numbers on the free surface, since other electrospinning parameters were not changed [17].

From Fig. $3 b$ it is clear that the uniformity index, $C_{F U}$, of the fiber distribution drops for a concentration increase from $11 \%$ to $12 \%$ and afterwards behaves unstably, demonstrating its minimum for $14 \%$. This means that $14 \%$ of electrospun material has the most monotonous/narrow fiber diameter distribution. Compared to the uniformity index reported by other researchers on different materials [16] and [36], it can be noted that all of the obtained values show very homogeneous distributions of fibers, since researchers report a uniformity index in the range of 1.1 to 1.3 to be narrow. Electrospinning voltage does not have any consistent effect on the fiber uniformity coefficient.

From the presented results in Figs. 1 and 3, it can be concluded that the polymer concentration and, consequently, viscosity play a more pronounced role in fiber creation compared to voltage. Similar findings can be found in the literature for both needleless and nozzle electrospinning [16], [37] and [38].

\subsection{Analysis of Defects}

During the fiber electrospinning at the maximal and minimal voltage settings $(75 \mathrm{kV}$ and $80 \mathrm{kV})$, defects in fibers were noted in some cases. Select examples are shown in SEM images in Fig. 4. These fibers were not used for the analysis of fiber diameter presented in the previous chapter.

Fig. 4a shows the formation of beads $-\mathrm{a}$ thick lump of material. This occurred only at the low voltage for the least concentrated solution. This phenomenon has been observed for needle electrospinning of PA6 by other researchers [11] and [14] and can be related to several different physical processes, for example, if the fiber is not thoroughly dried, the relaxation process in a solution can cause the formation of the bead at the moment of contact with the receiving electrode when the electrostatic force on the solution disappears. The occurrence of beads can harm the properties of products made from fibers. For example, when used for filtration, beads can obstruct the media flow.

Fig. $4 \mathrm{~b}$ shows the formation of very thin fibers (spider nets) between the thicker fibers. This occurred only at higher concentrations, but not regularly in all repetitions. The appearance of spider nets is related to an excessive amount of charges inside the solution. In our case, to the highly reactive polar solvent formic acid [13] and [39]. Another important factor is the strength of the electrostatic field that should be high enough to activate the additional charges in the solution [13], [39] and [40]. High values of relative humidity for volatile solvents reduce the speed of evaporation, and the solvent stays liquid longer, which allows the formation of thin fibers between the primary 

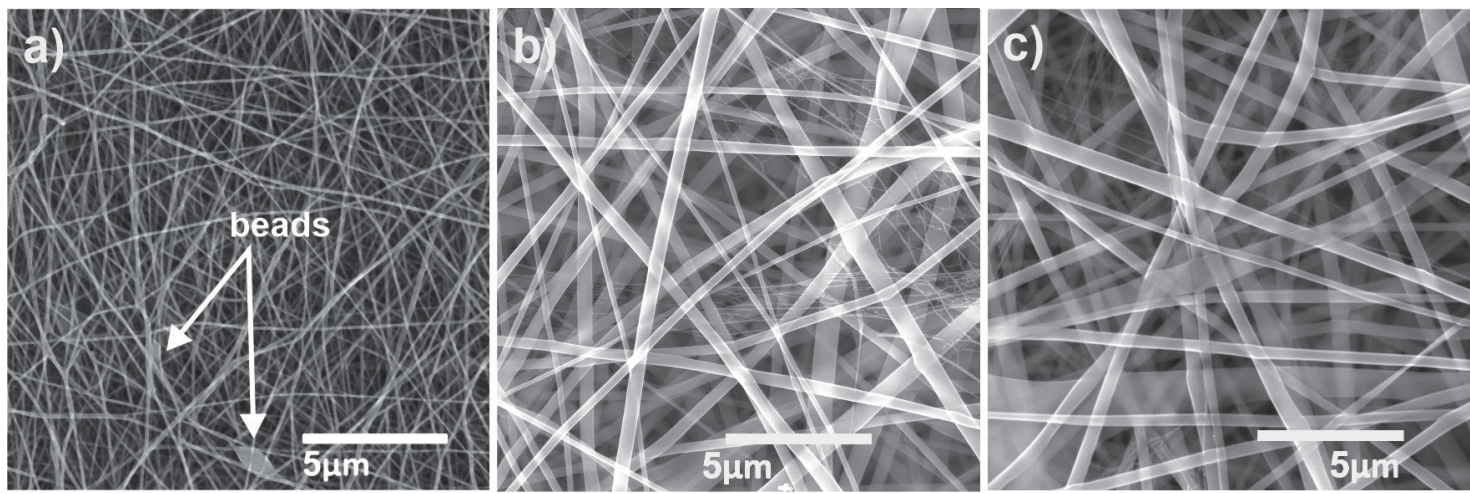

Fig. 4. Cases of defects on the electrospun mats;

a) beads, $11 \%$ PA6, $70 \mathrm{kV}$, b) spider nets, $14 \%$ PA6, $70 \mathrm{kV}$, and c) ribbon-like fibers, $13 \%$ PA6, $80 \mathrm{kV}$

fibers [39] and [40]. Spider nets can be desirable in some cases of filtration or scaffold applications since they can increase the filtration performance or cell proliferation. The exact mechanism of spider net formations, as well as their stable production, is still a matter of investigation.

Ribbon-like fibers, Fig. 4c, do not have direct negative or positive consequences, as was the case with the previous two irregularities. They appear due to a too low evaporation rate during electrospinning for concentrations higher than $13 \%$ and result in fibers with an uneven fiber diameter.

\section{CONCLUSIONS}

The paper investigates the effect of PA6 solution concentration and electrospinning voltage on the fiber diameter and diameter distribution manufactured with needleless electrospinning using the Nanospider setup. The main conclusions of our investigation are:

- Fiber diameter distribution appears to be generally independent of solution concentration and manufacturing conditions. All fibers exhibit a narrow fiber diameter distribution. The uniformity index of all investigated fibers is between 1.01 and 1.04, except for the most diluted solution with a uniformity index of approximately 1.06.

- Increasing solution concentration linearly increases the fiber diameter by about four times for all voltages.

- Changing the electrospinning voltage has a smaller effect. Increasing the voltage increases the fiber diameter, however, to a lesser extent (we measured a $35 \%$ diameter increase).

- This leads to the final conclusion. For robust changes of fiber diameter, it is preferable to change the PA6 solution concentration, since the solution concentration linearly increases with fiber diameter. On the other hand, for the precise control of fiber diameter, control of the electrospinning voltage should be used.

\section{ACKNOWLEDGEMENTS}

This work was financially supported by the Slovenian Research Agency as part of project L2-7550 and research core funding No. P2-0264 and partially financed by Fresenius Kabi Deutschland GmbH. The authors acknowledge the contributions of Angelina Vorontsova, Alexander Abramov, Yuri Salkovsky and other members of the research team of Education and Research Institute of Nanostructures and Biosystems, Saratov State University. The authors also would like to thank BASF Slovenia for the in-kind donation of material for research purposes and Anton Paar d.o.o., Slovenia, for providing a cup-cylinder measuring system for rheological characterization.

\section{REFERENCES}

[1] Knǐ̌ek, R., Karhánková, D., Bajzík, V., Jirsák, O. (2019). Lamination of nanofibre layers for clothing applications. Fibres and Textiles in Eastern Europe, vol. 27, no. 1, p. 16-21, DOI:10.5604/01.3001.0012.7503.

[2] Jun, I., Han, H.-S., Edwards, J.R., Jeon, H. (2018). Electrospun fibrous scaffolds for tissue engineering: Viewpoints on architecture and fabrication. International Journal of Molecular Sciences, vol. 19, no. 3, art. ID 745, D0l:10.3390/ ijms19030745.

[3] Lev, J., Holba, M., Došek, M., Kalhotka, L., Detvanová, L. (2013). Microfiltration nanofibrous membrane for wastewater treatment. NANOCON $5^{\text {th }}$ International conference, Brno.

[4] Aussawasathien, D., Teerawattananon, C., Vongachariya, A. (2008). Separation of micron to sub-micron particles from water: Electrospun nylon-6 nanofibrous membranes as pre- 
filters. Journal of Membrane Science, vol. 315, no. 1-2, p. 1119, D0I:10.1016/j.memsci.2008.01.049.

[5] Filatov, Y. N. (2001). Elektroformovanie voloknistyh materialov (Electroforming of fibrous materials process). Kirichenko, V.N. (ed.), Moscow.

[6] Wang, R., Liu, Y., Li, B., Hsiao, B.S., Chu, B. (2012). Electrospun nanofibrous membranes for high flux microfiltration. Journal of Membrane Science, vol. 392-393, p. 167-174, D0l:10.1016/j. memsci.2011.12.019.

[7] Reneker, D.H., Yarin, A.L. (2008). Electrospinning jets and polymer nanofibers. Polymer, vol. 49, no. 10, p. 2387-2425, DOI:10.1016/j.polymer.2008.02.002.

[8] Taylor, G.I. (1964). Disintegration of water drops in an electric field. Proceedings of the Royal Society A: Mathematical, Physical and Engineering Sciences, vol. 280, no. 1382, p. 383-397, D0I:10.1098/rspa.1964.0151.

[9] Yarin, A.L., Koombhongse, S., Reneker, D.H. (2001). Taylor cone and jetting from liquid droplets in electrospinning of nanofibers. Journal of Applied Physics, vol. 90, no. 9, p. 48364846, Dol:10.1063/1.1408260.

[10] Ding, B., Kim, H.-Y., Lee, S.-C., Lee, D.-R., Choi, K.-J. (2002). Preparation and characterization of nanoscaled polyvinyl alcohol fibers via electrospinning. Fibers and Polymers, vol. 3, no. 2, p. 73-79, DOl:10.1007/BF02875403.

[11] Mit-uppatham, C., Nithitanakul, M., Supaphol, P. (2004). Ultrafine electrospun polyamide- 6 fibers: Effect of solution conditions on morphology and average fiber diameter. Macromolecular Chemistry and Physics, vol. 205, no. 17, p. 2327-2338, DOl:10.1002/macp.200400225.

[12] Yalcinkaya, F., Yalcinkaya, B., Jirsak, O. (2016). Dependent and independent parameters of needleless electrospinning. Electrospinning - Material, Techniques, and Biomedical Applications, InTech, D0I:10.5772/65838.

[13] Nirmala, R., Nam, K.T., Park, S.J., Shin, Y.S., Navamathavan, R., Kim, H.Y. (2010). Formation of high aspect ratio polyamide- 6 nanofibers via electrically induced double layer during electrospinning. Applied Surface Science, vol. 256, no. 21, p. 6318-6323, D0I:10.1016/j.apsusc.2010.04.010.

[14] Heikkilä, P., Harlin, A. (2008). Parameter study of electrospinning of polyamide-6. European Polymer Journal, vol. 44, no. 10, p. 3067-3079, D0l:10.1016/j. eurpolymj.2008.06.032.

[15] Jirsak, O., Petrik, S. (2012). Recent advances in nanofibre technology: Needleless electrospinning. International Journal of Nanotechnology, vol. 9, no. 8-9, p. 836-845, D0l:10.1504/ IJNT.2012.046756.

[16] Yener, F., Jirsak, 0. (2012). Comparison between the needle and roller electrospinning of polyvinylbutyral, Journal of Nanomaterials, vol. 2012, no. February, DOI:10.1155/2012/839317.

[17] Yener, F., Jirsak, O. (2012). Development of new methods for study of mechanism of electrospinning. 19th International Conference Structure and Structural Mechanics of Textiles (Vol. 14), Liberec.

[18] Adomavičiute, E., Stanys, S., Banuškevičiute, A., Milašius, R. (2010). Influence of the shape of the bottom rotating electrode on the structure of Electrospun mats. Fibres and Textiles in Eastern Europe, vol. 18, no. 6, p. 49-53.
[19] BASF. (2013). Ultramid (PA). Product broshure, from https:// www.basf.com/documents/cn/en/chinaplas/Ultramid_ brochure.pdf, accessed 2017-09-06.

[20] Kraus, M.A., Frommer, M.A., Nemas, M., Gutman, R. (1976). Urea-rejecting membranes and their application in the development of a miniature artificial kidney. Journal of Membrane Science, vol. 1, p. 115-127, D0l:10.1016/S03767388(00)82262-0.

[21] Čapková, P., Čajka, A., Kolská, Z., Kormunda, M., Pavlík, J., Munzarová, M., Dopita, M., Rafaja, D. (2015). Phase composition and surface properties of nylon-6 nanofibers prepared by nanospider technology at various electrode distances. Journal of Polymer Research, vol. 22, no. 6, DOI:10.1007/s10965-015-0741-3.

[22] Yin, G., Zhao, Q., Zhao, Y., Yuan, Y., Yang, Y. (2013). The electrospun polyamide 6 nanofiber membranes used as high efficiency filter materials: Filtration potential, thermal treatment, and their continuous production. Journal of Applied Polymer Science, vol. 128, no. 2, p. 1061-1069, D0l:10.1002/ app.38211.

[23] Aulova, A., Cvenkel, A., Žakelj, S., Planinšek, O., Kristl, A., Emri, I. (2018). Mechanical properties and drug permeability of the PA6 membranes prepared by immersion precipitation from PA6 - formic acid - water system. Journal of Membrane Science, vol. 562, p. 67-75, Dol:10.1016/j.memsci.2018.05.022.

[24] Ryšánek, P., Malý, M., Čapková, P., Kormunda, M., Kolská, Z., Gryndler, M., Novák, O., Hocelíková, L., Bystrianský, L., Munzarová, M. (2017). Antibacterial modification of nylon-6 nanofibers: structure, properties and antibacterial activity. Journal of Polymer Research, vol. 24, D0l:10.1007/s10965017-1365-6.

[25] Ryklin, D.B., Jasinskaya, N.N., Evtushenko, A.V., Dzhumagulyev, D.D. (2016). Investigation of PA6 solution for electrospinning of nanofibrous coatings. Vestnik of Vitebsk State Technological University, vol. 1, p. 90-98.

[26] Pant, H.R., Bajgai, M.P., Yi, C., Nirmala, R., Nam, K.T., Baek, W., Kim, H.Y. (2010). Effect of successive electrospinning and the strength of hydrogen bond on the morphology of electrospun nylon-6 nanofibers. Colloids and Surfaces A: Physicochemical and Engineering Aspects, vol. 370, no. 1-3, p. 87-94, DOl:10.1016/j.colsurfa.2010.08.051.

[27] Ungur, G., Hrůza, J. (2017). Modified polyurethane nanofibers as antibacterial filters for air and water purification. RSC Advances, vol. 7, no. 78, p. 49177-49187, D0l:10.1039/ c7ra06317b.

[28] Formenti, S., Castagna, R., Momentè, R., Bertarelli, C., Briatico-Vangosa, F. (2016). The relevance of extensional rheology on electrospinning: The polyamide/iron chloride case. European Polymer Journal, vol. 75, p. 46-55, D0l:10.1016/j. eurpolymj.2015.12.003.

[29] Van Krevelen, D.W., Te Nijenhuis, K. (2009). Chapter 16 Rheological Properties of Polymer Solutions. Properties of Polymers, p. 599-644, D0l:10.1016/B978-0-08-0548197.00016-9.

[30] Wang, C., Cheng, Y.W., Hsu, C.H., Chien, H.S., Tsou, S.Y. (2011). How to manipulate the electrospinning jet with controlled properties to obtain uniform fibers with the smallest diameter? - A brief discussion of solution electrospinning 
process. Journal of Polymer Research, vol. 18, p. 111-123, DOI:10.1007/s10965-010-9397-1.

[31] Sill, T.J., von Recum, H.A. (2008). Electrospinning: Applications in drug delivery and tissue engineering. Biomaterials, vol. 29, no. 13, p. 1989-2006, D0l:10.1016/j. biomaterials.2008.01.011.

[32] Huang, C., Chen, S., Lai, C., Reneker, D.H., Qiu, H., Ye, Y., Hou, H. (2006). Electrospun polymer nanofibres with small diameters. Nanotechnology, vol. 17, no. 6, p. 1558, DOI:10.1088/0957-4484/17/6/004.

[33] Guerrini, L.M., Branciforti, M.C., Canova, T., Bretas, R.E.S. (2009). Electrospinning and characterization of polyamide 66 nanofibers with different molecular weights. Materials Research, vol. 12, no. 2, p. 181-190, D0l:10.1590/S151614392009000200012.

[34] Wang, R., Liu, Y., Li, B., Hsiao, B.S., Chu, B. (2012). Electrospun nanofibrous membranes for high flux microfiltration. Journal of Membrane Science, vol. 392-393, p. 167-174, D0I:10.1016/j. memsci.2011.12.019.

[35] Ahn, Y.C., Park, S.K., Kim, G.T., Hwang, Y.J., Lee, C.G., Shin, H.S., Lee, J.K. (2006). Development of high efficiency nanofilters made of nanofibers. Current Applied Physics, vol. 6, no. 6, p. 1030-1035, DOl:10.1016/j.cap.2005.07.013.
[36] Cengiz Çallioğlu, F. (2014). The effect of glyoxal cross-linker and $\mathrm{NaCl}$ Salt addition on the roller Electrospinning of poly(vinyl alcohol) nanofibers. Tekstil ve Konfeksiyon, vol. 24, no. 1, p. 15-20, D0l:10.32710/tekstilvekonfeksiyon.621380.

[37] Pupkevičiute, S., Adomavičiute, E., Stanys, S. (2013). Analysis of structure of electrospun nonwoven mats from pure PCL nano/micro fibres. Materials Science, vol. 19, no. 3, p. 295300, DOI:10.5755/j01.ms.19.3.1420.

[38] Simanenkova, L.M., Kil'deeva, N.R. (2012). Electrospinning of ultrathin fibers from acetic-acid solutions of a polyelectrolyte containing amines. Fibre Chemistry, vol. 44, no. 4, p. 232 235, DOl:10.1007/s10692-012-9436-2.

[39] Ding, B., Li, C., Miyauchi, Y., Kuwaki, O., Shiratori, S. (2006). Formation of novel 2D polymer nanowebs via electrospinning. Nanotechnology, vol. 17, no. 15, p. 3685-3691, DOI:10.1088/0957-4484/17/15/011.

[40] Wang, N., Wang, X., Ding, B., Yu, J., Sun, G. (2012). Tunable fabrication of three-dimensional polyamide-66 nano-fiber/ nets for high efficiency fine particulate filtration. Journal of Materials Chemistry, vol. 22, no. 4, p.1445-1452, DOI:10.1039/c1jm14299b. 